\title{
The Gravitational Radiation Emitted by Two Quasi-Particles around a Schwarzschild Black Hole
}

\author{
Amos S. Kubeka \\ Department of Mathematical Sciences, University of South Africa, Pretoria, South Africa \\ Email: kubekas@unisa.ac.za
}

How to cite this paper: Kubeka, A.S. (2018) The Gravitational Radiation Emitted by Two Quasi-Particles around a Schwarzschild Black Hole. Journal of Modern Physics, 9, 1195-1214. https://doi.org/10.4236/jmp.2018.96072

Received: July 19, 2017

Accepted: May 12, 2018

Published: May 15, 2018

Copyright $\odot 2018$ by author and Scientific Research Publishing Inc. This work is licensed under the Creative Commons Attribution International License (CC BY 4.0).

http://creativecommons.org/licenses/by/4.0/

\section{c) (i) Open Access}

\begin{abstract}
We model analytically a relativistic problem consisting of two quasi-particles each with mass $m$ in close orbit around a static Schwarzschild black hole with mass $M=1$ situated at the center of mass of the system. The angular momentum $l$ of the system is taken to be 2 . We model the mass density of the orbiting particles as a $\delta$-function and we assume that there are no deformations. To model the system, we apply the second-order differential equation obtained elsewhere for a dynamic thin matter shell on a Schwarzschild background. As it is the case in this paper, the framework on which the equation was obtained is Bodi-Sachs. The only change in the equation is that now the quasi-normal mode parameter represents the particle's orbital frequency from which we are able to analytically compute the gravitational radiation emitted by the system at null infinity. We note that in a real astrophysical scenario the dynamics of the particles paths will be very dynamic and complicated and that the analytical methods used here will have to be developed further to accommodate that.
\end{abstract}

\section{Keywords}

Gravitational Radiation, Schwarzschild Black Hole, Quasi-Particles, Quasi-Normal Mode Null Formalism

\section{Introduction}

Until recently, all sorts of relativistic binary systems have been studied only theoretically and on the 14 September 2015 a team of LIGO and Virgo collaborators announced their first detection of a gravitational wave signal from a binary black hole system of about 36 and 29 solar masses. This announcement reaffirmed the predictions of the existance of gravitational waves as predicted by 
GR and most importantly the affirmation that indeed binary relativistic systems do exist in nature. LIGO, Virgo and all other promising gravitational wave detectors will thus provide with the means to be able to detect all kinds of relativistic binary systems with all sorts of physical properties [1]-[23].

More so, the works in this direction have been in the realm of numerical relativity with a special focus of relativistic two body problems i.e. Black hole-black hole binary, black hole-neutron star binary, and neutron star-neutron star binary. The case of a three-body problem as it is the case here has never been studied before either numerically or analytically even though there is a much compelling possibility that in very dense cluster of galaxies these kinds of systems could in fact be found in the near future. As it was the case at the beginning of the research work in relativistic binary systems in the past decades, it is also very likely that there will be arising technical and computational issues for the gravitating three body problem in full numerical relativity. The analytic method used in this paper has been used by the author in [24] to validate other analytical results obtained by [25] [26] for a binary system.

In the setting of this paper, we use the analytical results by Bishop [27] to study analytically the relativistic triple system consisting of two point-particles in quasi-orbit around a static Schwarzschild black hole. In practice the particles could be either two black holes, two neutron stars or in another setting a combination of both. Our objective thus is to determine the amount of the emitted gravitational radiation by the system at $\mathcal{I}^{+}$in Bondi-Sachs formalism. This paper is structured as follows: Section 2 gives the background material. Section 3 defines the physical problem to be studied. Section 4 calculates the emitted gravitational radiation at $\mathcal{I}^{+}$.

\section{Background}

\subsection{Formalism}

The Bondi-Sachs formalism uses coordinates $x^{i}=\left(u, r, x^{A}\right)$ based upon a family of outgoing null hypersurfaces. We label these hypersurfaces by $u=$ const., null rays by $x^{A} \quad\left(A=2,3, x^{2}=\theta, x^{3}=\phi\right)$, and the surface area coordinate by $r$. In this coordinates system the Bondi-Sachs metric [28] [29] takes the form

$$
\begin{aligned}
\mathrm{d} s^{2}= & -\left[\mathrm{e}^{2 \beta}\left(1+\frac{W}{r}\right)-r^{2} h_{A B} U^{A} U^{B}\right] \mathrm{d} u^{2}-2 \mathrm{e}^{2 \beta} \mathrm{d} u \mathrm{~d} r \\
& -2 r^{2} h_{A B} U^{B} \mathrm{~d} u \mathrm{~d} x^{A}+r^{2} h_{A B} \mathrm{~d} x^{A} \mathrm{~d} x^{B},
\end{aligned}
$$

where $h^{A B} h_{B C}=\delta_{B}^{A}$ and $\operatorname{det}\left(h_{A B}\right)=\operatorname{det}\left(q_{A B}\right)$, with $q_{A B}$ being a unit sphere metric, $U$ is the spin-weighted field given by $U=U^{A} q_{A}$. For a Schwarzschild space-time, $W=-2 M$. We define the complex quantity $J$ by

$$
J=q^{A} q^{B} h_{A B} / 2 .
$$

For the Schwarzschild space-time, we have $J$ and $U$ being zero and thus they can be regarded as a measure of the deviation from spherical symmetry, and in 
addition, they contain all the dynamic content of the gravitational field in the linearized regime [30]. Usually we can describe this space-time by $\beta=0$ and $W=-2 M$, or by $\beta=\beta_{c}$ (constant) and $W=\left(\mathrm{e}^{2 \beta_{c}}-1\right) r-2 M$.

For spherical harmonics we use ${ }_{s} Z_{l m}$ rather than ${ }_{s} Y_{l m}$ as basis functions as follows [27]

$$
\begin{array}{ll}
{ }_{s} Z_{l m}=\frac{1}{\sqrt{2}}\left[{ }_{s} Y_{l m}+(-1)^{m}{ }_{s} Y_{l-m}\right] & \text { for } m>0 \\
{ }_{s} Z_{l m}=\frac{i}{\sqrt{2}}\left[(-1)^{m}{ }_{s} Y_{l m}-{ }_{s} Y_{l-m}\right] & \text { for } m>0 \\
{ }_{s} Z_{l 0}={ }_{s} Y_{l 0}, &
\end{array}
$$

The $s=0$ will be omitted in the case $s=0$, i.e. $Z_{l m}={ }_{0} Z_{l m}$. The ${ }_{s} Z_{l m}$ are orthonormal and real. We assume the following ansatz

$$
\begin{aligned}
& J=\operatorname{Re}\left(J_{0}(r) \mathrm{e}^{i \sigma u}\right) \partial^{2} Z_{l m}, \quad U=\operatorname{Re}\left(U_{0}(r) \mathrm{e}^{i \sigma u}\right) \partial Z_{l m}, \\
& \beta=\operatorname{Re}\left(\beta_{0}(r) \mathrm{e}^{i \sigma u}\right) Z_{l m}, \quad \omega=\operatorname{Re}\left(\omega_{0}(r) \mathrm{e}^{i \sigma u}\right) Z_{l m},
\end{aligned}
$$

where $r_{0}$ is the position of the matter shell, and $\sigma$ the complex frequency mode which is physical damped and which further means that $\operatorname{Im}(\sigma)>0$. In the Bondi frame, the field equations splits into;

- the hypersurface equations and the evolution equations given by

$$
\begin{aligned}
& R_{r r}: \frac{4}{r} \beta_{, r}=8 \pi T_{r r} \\
& q^{A} R_{r A}: \frac{1}{2 r}\left(4 ð \beta-2 r ð \beta_{, r}+r \bar{\varnothing} J_{, r}+r^{3} U_{, r r}+4 r^{2} U_{, r}\right)=8 \pi q^{A} T_{r A} \\
& h^{A B} R_{A B}:(4-2 \partial \bar{\partial}) \beta+\frac{1}{2}\left(\bar{ळ}^{2} J+\partial^{2} \bar{J}\right)+\frac{1}{2 r^{2}}\left(r^{4} \check{\partial} \bar{U}+r^{4} \bar{\partial} U\right)_{, r}-2 \omega_{, r} \\
& =8 \pi\left(h^{A B} T_{A B}-r^{2} T\right) \\
& q^{A} q^{B} R_{A B}:-2 \partial^{2} \beta+\left(r^{2} \partial U\right)_{, r}-2(r-M) J_{, r}-\left(1-\frac{2 M}{r}\right) r^{2} J_{, r r}+2 r(r J)_{, u r} \\
& =8 \pi q^{A} q^{B} T_{A B},
\end{aligned}
$$

- and the constraint equations for off the matter shell in the case of vacuum given by

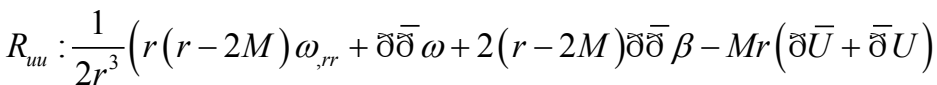

$$
\begin{aligned}
& \left.-r^{3}(\check{\partial} \bar{U} \bar{\partial} U)_{, u}+2 r \omega_{, u}\right)=0, \\
& R_{u r}: \frac{1}{4 r^{2}}\left(2 r \omega_{, r r}+4 ð \bar{\varnothing} \beta-\left(r^{2} \nsim \bar{U}+r^{2} \bar{\varnothing} U\right)_{, r}\right)=0, \\
& q^{A} R_{u A}: \frac{1}{4 r^{2}}\left(2 r ð \omega_{, r}-2 ð \omega+2 r^{2}(r-2 M)\left(4 U_{, r}+r U_{, r r}\right)+4 r^{2} U\right. \\
& \left.+r^{2}\left(ð \bar{\partial} U-\check{\partial}^{2} \bar{U}\right)+2 r^{2} \bar{\partial} J_{, u}-2 r^{4} U_{, u r}-4 r^{2} \partial \beta_{, u}\right)=0,
\end{aligned}
$$


Ref. [27] got the following second order differential equation when solving the above systems of ordinary differential equations for the Schwarzschild background;

$$
\begin{aligned}
& x^{3}(1-2 x M) \frac{\mathrm{d}^{2} J_{2}}{\mathrm{~d} x^{2}}+2 \frac{\mathrm{d} J_{2}}{\mathrm{~d} x}\left(2 x^{2}+i \sigma x-7 x^{3} M\right) \\
& -2\left(x\left(l^{2}+l-2\right) / 2+8 M x^{2}+i \sigma\right) J_{2}=0
\end{aligned}
$$

where $J_{2}(x) \equiv \mathrm{d}^{2} J_{0+} / \mathrm{d} x^{2}$ and $x=1 / r, x$ is the compactification factor in this language. Bishop et al. [31] solved Equation (12) numerically and obtained interesting quasi-normal modes results of a Schwarzschild white hole. However in this paper, we are going to solved it for a different problem since we can apply the same physical settings in the Bondi-frame to model our problem with $\sigma$ having a different physical meaning as we shall see later.

\subsection{An analytic Algorithm for Calculating the Gravitational News}

We shall use the following algorithm to calculate the gravitational radiation from the system.

- First we use Equation (12) and the constraints Equations (9)-(11) to get the junction conditions for the Bondi-Sachs matric variables $U, \omega$ and $J$ at the boundary i.e. shell,

- Second we test if $J, J_{, r}, U, U_{, r}$, and $\omega$ are smooth across the boundary and if this is true, we then

- Calculate the News function at $\mathcal{I}^{+}$.

\section{The Problem}

We consider a system consisting of two point-particles with equal mass $m$ in quasi-orbit around a stationary Schwarzschild black hole with mass $M$ situated at the center of mass $r$ of the particles when $l$ is 2 . We take the orbital radius to be at $r_{0}$ which means that the distance between the particles is $2 r_{0}$. We take the initial position of particle 1 to be at $r_{0}$ with $\theta$ and $\phi$ given by $\pi / 2$ and $v u$ respectively, $v$ is the orbital frequency and $u$ the orbital period of the particles. We also take the initial position of a particle 2 to be at $r_{0}$ with $\theta$ and $\phi$ given by $\pi / 2$ and $v u+\pi$ respectively. This imply that the rotation in the following figure is in the $y z$ plane. The initial positions of the objects on the figure should not be confused with the actual initial positions just outline which in actual sense should be along the $y$ axis with the particle 1 on the right and the particle 2 on the left.

The dynamics of this problem is governed by Equation (12) and for our numerical calculation purposes we shall use its Ricatti form [31]

$$
\frac{\mathrm{d} v}{\mathrm{~d} x}=1+\frac{2 v}{x^{2}(1-2 x)}\left((x-v)\left(2+\frac{i v}{x}\right)-x(7 x+8 v)\right)
$$

where $v$ is the orbital period of the system. 


\section{The Emitted Gravitational Radiation}

\subsection{The Linear Expansion of the Light Rays From the System to $\mathcal{I}^{+}$}

We model the problem as follows, we start by applying Equation (5) with $T_{r r}$ given by

$$
\rho\left(1-\frac{2 M}{r}\right)^{-1}
$$

where the matter density $\rho$ in the background space-time is given by

$$
\rho=\frac{\left(1-\frac{2 M}{r}\right)^{-1}}{r_{0}^{2}} \delta\left(r-r_{0}\right)\left(\theta-\frac{\pi}{2}\right)[\delta(\phi-v u)+\delta(\phi-v u-\pi)] .
$$

Inside the particles orbital radius $r<r_{0}$ we set

$$
\beta=0 \text {, }
$$

and outside the particles orbital radius $r>r_{0}$ we set

$$
\beta=\Sigma_{l m} \beta_{l m} Z_{l m} \text {. }
$$

Now integrating with respect to $r$ we get

$$
\Sigma_{l m} \beta_{l m} Z_{l m}=\frac{2 \pi}{r_{0}}\left(1-\frac{2 M}{r}\right)^{-2} \delta\left(\theta-\frac{\pi}{2}\right)[\delta(\phi-v u)+\delta(\phi-v u-\pi)]
$$

By multiplying Equation (18) with $Z_{l^{\prime} m^{\prime}}$ we get

$$
Z_{l^{\prime} m^{\prime}} \Sigma_{l m} \beta_{l m} Z_{l m}=\frac{2 \pi}{r_{0}}\left(1-\frac{2 M}{r}\right)^{-2} \delta\left(\theta-\frac{\pi}{2}\right)\left[Z_{l^{\prime} m^{\prime}} \delta(\phi-v u)+Z_{l^{\prime} m^{\prime}} \delta(\phi-v u-\pi)\right]
$$

and integrating over the sphere it simplifies to

$$
\beta_{l^{\prime} m^{\prime}}=\frac{2 \pi}{r_{0}}\left(1-\frac{2 M}{r}\right)^{-2}\left[Z_{l^{\prime} m^{\prime}}\left(\frac{\pi}{2}, v u\right)+Z_{l^{\prime} m^{\prime}}\left(\frac{\pi}{2}, v u+\pi\right)\right] .
$$

From Equation (20), for $m^{\prime} \neq 0$ we the gravitational radiation otherwise we don't, and that $\beta_{l^{\prime} m^{\prime}}$ are generally non-zero for even $l$ and $m^{\prime}$. We now consider the case $l^{\prime}=2$ and we note that

$$
\begin{aligned}
& \beta_{21}=0, \\
& \beta_{2,-1}=0,
\end{aligned}
$$

and that

$$
\beta_{20} \neq 0 \text {. }
$$

We note that $\beta_{20}$ mode does not vary in time and hence it does not contain the emitted gravitational radiation. Thus we are only interested in $\beta_{22}$ and $\beta_{2,-2}$ modes. We use the following normalized spherical harmonics

$$
\begin{aligned}
& Y_{22}=\frac{1}{4} \sqrt{\frac{15}{2 \pi}} \sin ^{2} \theta \mathrm{e}^{2 i \phi}, \\
& Y_{2,-2}=\frac{1}{4} \sqrt{\frac{15}{2 \pi}} \sin ^{2} \theta \mathrm{e}^{-2 i \phi},
\end{aligned}
$$


and the fact that

$$
\begin{aligned}
& Z_{22}=\frac{1}{\sqrt{2}}\left(Y_{22}+Y_{2,-2}\right), \\
& Z_{2,-2}=\frac{i}{\sqrt{2}}\left(Y_{2,-2}-Y_{22}\right),
\end{aligned}
$$

to get

$$
Z_{22}=\frac{\sqrt{2}}{4} \sqrt{\frac{15}{2 \pi}} \sin ^{2} \theta \cos 2 \phi
$$

and

$$
Z_{2,-2}=\frac{\sqrt{2}}{4} \sqrt{\frac{15}{2 \pi}} \sin ^{2} \theta \sin 2 \phi .
$$

Thus from Equation (20)

$$
\begin{aligned}
\beta_{22} & =\frac{2 \pi}{r_{0}}\left(1-\frac{2 M}{r_{0}}\right)^{-2} \frac{\sqrt{2}}{4} \sqrt{\frac{15}{2 \pi}} \cos (2 v u) 2 \\
& =\frac{\sqrt{15 \pi}}{r_{0}}\left(1-\frac{2 M}{r_{0}}\right)^{-2} \cos (2 v u),
\end{aligned}
$$

and similarly

$$
\beta_{2,-2}=\frac{\sqrt{15 \pi}}{r_{0}}\left(1-\frac{2 M}{r_{0}}\right)^{-2} \sin (2 v u)
$$

and then finally we write

$$
\beta=\frac{\sqrt{15 \pi}}{r_{0}}\left(1-\frac{2 M}{r_{0}}\right)^{-2}\left(\operatorname{Re}\left\{\mathrm{e}^{2 i v u}\right\} Z_{22}+\operatorname{Re}\left\{-i \mathrm{e}^{2 i v u}\right\} Z_{2,-2}\right)
$$

Now taking $M=1$, Equation (32) then becomes

$$
\beta=\frac{\sqrt{15 \pi}}{r_{0}}\left(1-\frac{2}{r_{0}}\right)^{-2}\left(\operatorname{Re}\left\{\mathrm{e}^{2 i v u}\right\} Z_{22}+\operatorname{Re}\left\{-i \mathrm{e}^{2 i v u}\right\} Z_{2,-2}\right)
$$

\subsection{The Gravitational Radiation}

We assume that the orbit is at the innermost stable circular orbit (ISCO), so that $r=r_{0}=6$. We then found the change in the Schwarzschild coordinate time $\mathcal{T}$ for one complete revolution of 92.3436 from which we found the orbital frequency $v$ of 0.0680 .

To now find the numerical solutions to continue Equation (13) we make the spatial coordinate transformation of $x=1 / r$ which then imply that the ISCO is now at $x_{m n}=1 / 6$. The numerical computations are done in the domains

$$
D_{+}=\left\{0<x<x_{m n}\right\} \text { and } D_{-}=\left\{x_{m n}<x<0.5\right\},
$$

with numerical solutions $v_{+}(x)$ and $v_{-}(x)$ respectively. We start the calculation with the transformed Equation (12) given by 


$$
\begin{aligned}
U(x)= & 2 \beta_{0} x-\frac{1}{2} x^{4}(1-2 x M) \frac{\mathrm{d}^{3}}{\mathrm{~d} x^{3}} J(x)-x^{3}\left(x-2 x^{2} M+i v\right) \frac{\mathrm{d}^{2}}{\mathrm{~d} x^{2}} J(x) \\
& +x\left(2 x+2 x^{2} M+i v\right) \frac{\mathrm{d}}{\mathrm{d} x} J(x)-i v J(x)
\end{aligned}
$$

where $U_{+}(x), U_{-}(x)$ are the Bondi metric functions, and $\beta_{0+}, \beta_{0-}$ are the values of the expansion of the light rays $\beta$ given by Equation (32) in the exterior and interior domains respectively. Bishop [27] has indicated that the derivatives of $J$ should not be worked out numerically, but should be worked out analytically in terms of $J_{1}, J_{2}$ and $v$ from Equation (13) with $v=0.0680$.

We define the general solutions for $J_{2}(x)$ at $x_{m n}$ outside and inside the orbital radius respectively as

$$
\begin{aligned}
& J_{+}(x)=c_{4}+c_{1} x+c_{2} J_{0+}(x), \\
& J_{-}(x)=c_{9}+c_{6} x+c_{7} J_{0-}(x),
\end{aligned}
$$

where $c_{4}, c_{1}, c_{2}, c_{9}, c_{6}$ and $c_{7}$ are constants to be determined numerically. The functions $J_{0+}(x)$ and $J_{0-}(x)$ are analytic near $x_{m n}$ and therefore can be Taylor expand as

$$
\begin{aligned}
J_{+}(x)= & J_{0+}\left(x_{m n}\right)+\left(x-x_{m n}\right) \frac{\mathrm{d}}{\mathrm{d} x} J_{0+}(x)+\frac{\left(x-x_{m n}\right)^{2}}{2} \frac{\mathrm{d}^{2}}{\mathrm{~d} x} J_{0+}(x) \\
& +\frac{\left(x-x_{m n}\right)^{3}}{6} \frac{\mathrm{d}^{3}}{\mathrm{~d} x} J_{0+}(x), \\
J_{-}(x)= & J_{0-}\left(x_{m n}\right)+\left(x-x_{m n}\right) \frac{\mathrm{d}}{\mathrm{d} x} J_{0-}(x)+\frac{\left(x-x_{m n}\right)^{2}}{2} \frac{\mathrm{d}^{2}}{\mathrm{~d} x} J_{0-}(x) \\
& +\frac{\left(x-x_{m n}\right)^{3}}{6} \frac{\mathrm{d}^{3}}{\mathrm{~d} x} J_{0-}(x),
\end{aligned}
$$

which then results in Equations (36) and (39) being analytic near $x_{m n}$. We used Matlab ode45 solver to find numerical solutions of the above derivatives in Equations (38) and (39). We used stringent numerical conditions to get the results to about seven significant figures with RelTol of $10^{-12}$, AbsTol of $10^{-12}$, and the MaxStep of $0.2 \times 10^{-5}$ and the results we found to be

$$
\begin{gathered}
\frac{\mathrm{d}}{\mathrm{d} x} J_{0+}(x)=29144-2.280672 \times 10^{5} i, \\
\frac{\mathrm{d}^{2}}{\mathrm{~d} x} J_{0+}(x)=2.865551 \times 10^{6}-1.52335130 \times 10^{7} i, \\
\frac{\mathrm{d}^{3}}{\mathrm{~d} x} J_{0+}(x)=4.8870 \times 10^{7}-1.8591431 \times 10^{9} i,
\end{gathered}
$$

and

$$
\begin{gathered}
\frac{\mathrm{d}}{\mathrm{d} x} J_{0-}(x)=13.04337-1.31529 i, \\
\frac{\mathrm{d}^{2}}{\mathrm{~d} x} J_{0-}(x)=1.54689 \times 10^{2}-3.19980 \times 10^{1} i,
\end{gathered}
$$




$$
\frac{\mathrm{d}^{3}}{\mathrm{~d} x} J_{0-}(x)=-1.12428 \times 10^{3}-1.25311 \times 10^{3} i .
$$

We have tested for the consistency of the above results by using other Matlab solvers; ode23 and ode15s (which uses the Gears method i.e. backward differentiation formulas) and also observed the accuracy of about 15 significant figures. We went further with the test using ode23t which uses the trapezoidal rule, ode23s which is a modified Rosenbrock formula of order 2, and ode23tb which is an implicit Runge Kutta as opposed to ode45 and ode23 and found the consistency of about 8 significant figures and as opposed to 15 significant figures which is also accurate enough. This illustrate how accurate and valid the results are. These results are very crucial in obtaining the emitted gravitational radiation and hence determining the extent of their convergence is of most paramount importance.

From the hypersurface equation Equation (7) rewritten as

$$
-2 x^{2} \omega_{, x}=2\left(2-L_{2}\right) \beta_{0}+L_{2}\left(L_{2}+2\right) J-x^{4}\left(x^{-4} L_{2} U\right)_{, x}
$$

we are able to the Bondi metric function $\omega_{+}(r)$ and $\omega_{-}(r)$. But to find the solution the integration should be done analytically where possible. We only need a solution which is valid in a neighborhood of $x=x_{0}$. Henceforth, it is convenient to make the coordinate transformation $x \rightarrow r=1 / x$. Equation (46) can further be rewritten as

$$
2\left(2-L_{2}\right) \beta_{0}+L_{2}\left(L_{2}+2\right) J+\frac{1}{r^{2}}\left(r^{4} L_{2} U\right)_{, r}=2 \omega_{, r}
$$

where for $l=2$ we have $L_{2}=-6$. The constraints equations Equations (9), (10), and (11) now simplifies to

$$
\begin{aligned}
R_{u u}: & \frac{1}{2 r^{3}}\left(\left(r^{2}-2 M r\right) \omega_{, r r}-6 \omega-12(r-2 M) \beta_{0}+12 M r U\right. \\
& \left.-4 r(r-2 M) i v \beta_{0}+12 r^{3} i v U+2 r i v \omega\right)=0, \\
q^{A} R_{u A}: & \frac{1}{2 r^{2}}\left(r \omega_{, r}-\omega+4 r^{3} U_{, r}+r^{4} U_{, r r}+2 r^{2} U-2 M r^{3} U_{, r r}-8 M r^{2} U_{, r}\right. \\
& \left.-r^{2} i v J-r^{4} i v U_{, r}-2 r^{2} i v \beta_{0}\right)=0 .
\end{aligned}
$$

which we then apply in the domains $D_{+}$and $D_{-}$. Since these constraints are not completely analytic, this means that we should only evaluate them at the ISCO. We use them among others to eliminate the constants $c_{1}, c_{2}, c_{6}$, and $c_{7}$. We now assume that we end up with the solutions

$$
\omega_{+}(x)=c_{5}+\omega_{0+}(x), \omega_{-}(x)=c_{10}+\omega_{0-}(x), \text { with } \omega_{0+}\left(x_{0}\right)=\omega_{0-}\left(x_{0}\right)=0 .
$$

Thus, from the constraints $R_{u u-}\left(r_{0}\right), R_{u u+}\left(r_{0}\right), R_{u r-}\left(r_{0}\right), R_{u r+}\left(r_{0}\right)$, $q^{A} R_{u A-}\left(r_{0}\right), q^{A} R_{u A+}\left(r_{0}\right)$, and the hypersurface Equation (47), we found the metric variables $U_{+}\left(r_{0}\right), U_{-}\left(r_{0}\right), \omega_{+}\left(r_{0}\right)$, and $\omega_{-}\left(r_{0}\right)$. From which the expressions of the constants $c_{9}, c_{7}, c_{5}$, and $c_{10}$, were found.

We now impose the Bondi gauge conditions: 


$$
\beta_{0+}=0, \quad c_{4}=0,
$$

which means that for large $r, \beta_{0+}=0$ at $\mathcal{I}^{+}$imply that the coordinate time is the same as proper time and that the regularity at $\mathcal{I}^{+}$require $c_{4}=0$. We also impose the following junction conditions at $r_{0}$ :

$$
\begin{gathered}
J_{+}\left(r_{0}\right)=J_{-}\left(r_{0}\right), \\
2 U_{+}\left(r_{0}\right)=U_{-}\left(r_{0}\right), \\
\beta_{0-}=-2 \pi r_{0} \rho\left(1-\frac{2 M}{r_{0}}\right)^{-1} \\
\omega_{+}\left(r_{0}\right)-\omega_{-}\left(r_{0}\right)=-4 \pi r^{2} \rho .
\end{gathered}
$$

From the junction conditions, we were able to find the exact numerical values of the constants $c_{1}, c_{2}$, and $c_{6}$ at $r_{0}=6$. The exact numerical values of the constants $c_{9}, c_{7}, c_{5}$, and $c_{10}$ were then found by substituting the values of $c_{1}, c_{2}$, and $c_{6}$ back into their expressions. From here we were then able to plot the graphs of the Bondi metric functions $J_{-}\left(r_{0}\right), J_{+}\left(r_{0}\right), U_{-}\left(r_{0}\right), U_{+}\left(r_{0}\right)$, $\omega_{-}\left(r_{0}\right)$, and $\omega_{+}\left(r_{0}\right)$ as observed in the following graphs.

Physically the metric functions $J$ and $U$ have the smooth asymptotic expansion characteristic through out the entire computational domain and this property is confirmed in Figure 1 and Figure 2. The metric function $\omega$ do not have this physical property as can be confirmed in Figure 3 but this function is crucial in the calculation procedure of the gravitation radiation in the entire domain. Physically the function $J$ in the only one that have the time derivative and thus carries the gravitational radiation information to calculated at $\mathcal{I}^{+}$ and that all the other Bondi metric functions are intergrated radially from $\Gamma$ to $\mathcal{I}^{+}$. The above results indicate that the junction conditions at $r_{0}=6$ where

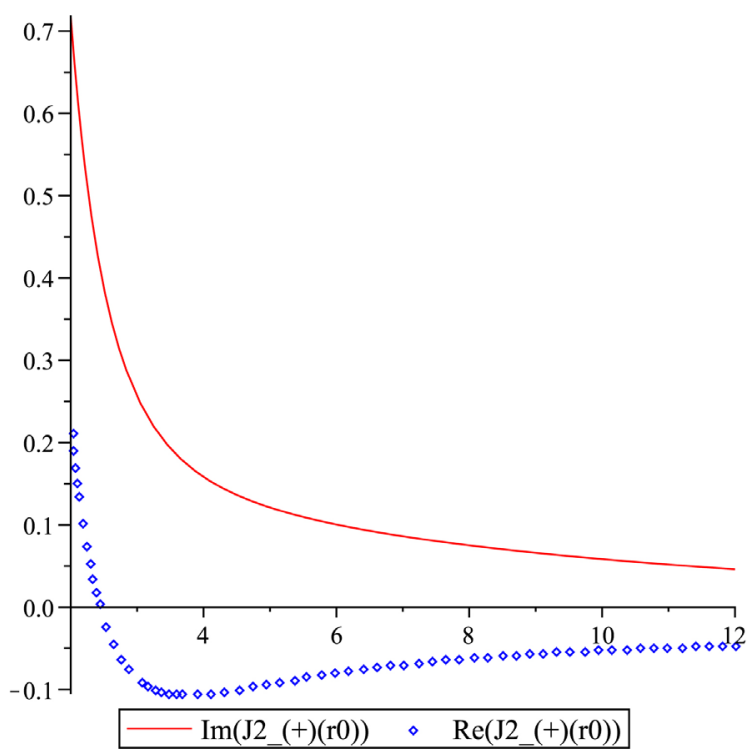

Figure 1. The graph of $\operatorname{Im}\left(J 2_{+}\left(r_{0}\right)\right)$ for $\operatorname{Im}(J)$ in the entire domain, and $\operatorname{Re}\left(J 2_{+}\right)$ for $\operatorname{Re}(J)$ also in the entire for the Schwarzschild space-time. $v=0.07$ and $\ell=2$. 


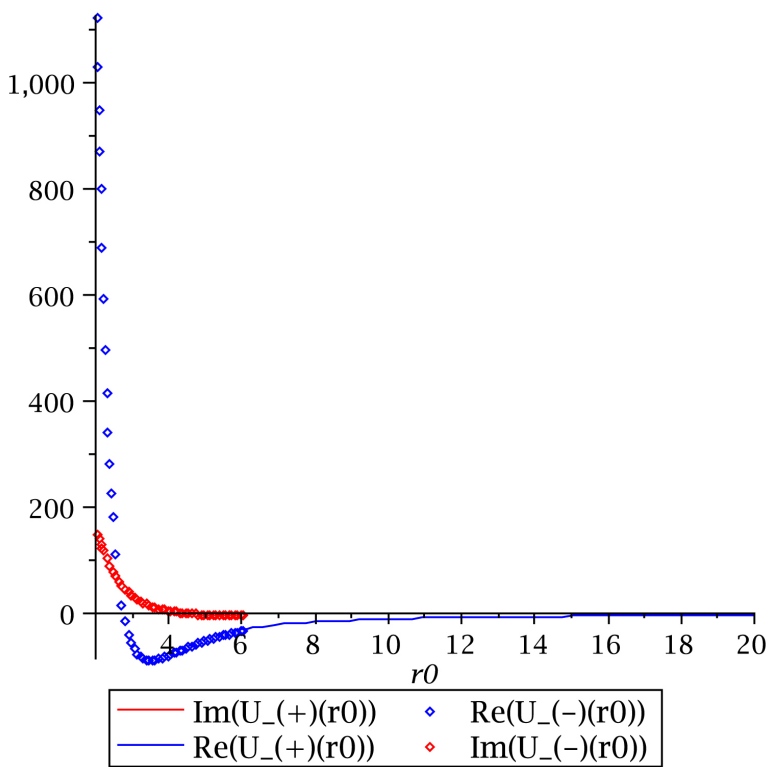

Figure 2. The graph of $\operatorname{Re}\left(U_{-}\left(r_{0}\right)\right), \operatorname{Im}\left(U_{-}\left(r_{0}\right)\right)$ and $\operatorname{Re}\left(U_{+}\left(r_{0}\right)\right), \operatorname{Im}\left(U_{+}\left(r_{0}\right)\right)$ for the Schwarzschild space-time. $v=0.07$ and $\ell=2$.

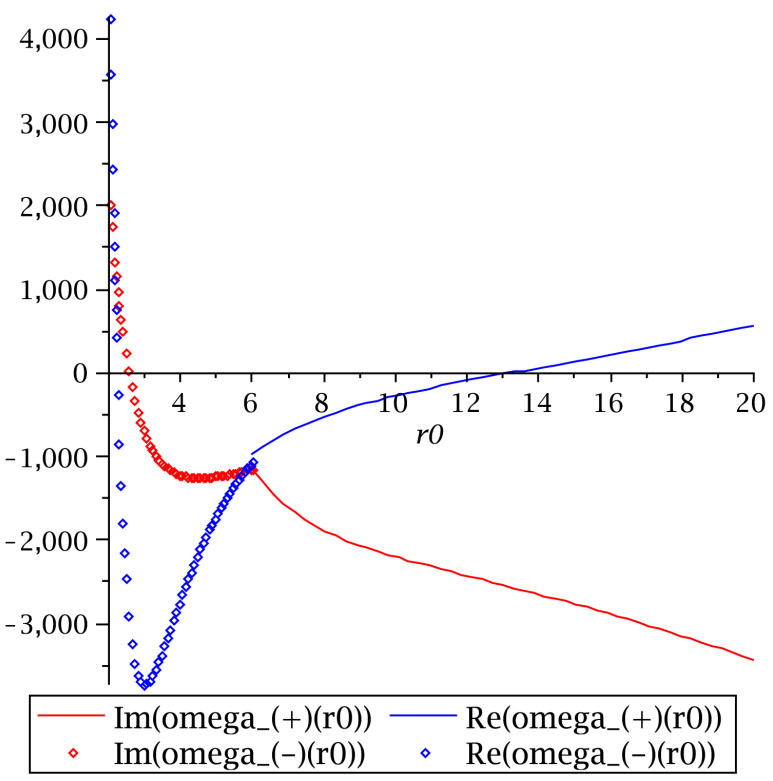

Figure 3. The graph of $\operatorname{Re}\left(\omega_{-}\left(r_{0}\right)\right), \operatorname{Im}\left(\omega_{-}\left(r_{0}\right)\right)$ and $\operatorname{Re}\left(\omega_{+}\left(r_{0}\right)\right), \operatorname{Im}\left(\omega_{+}\left(r_{0}\right)\right)$ for the Schwarzschild space-time. $v=0.07$ and $\ell=2$.

implemented correctly and that our numerical methods and the analytical algorithms we implemented to calculating the gravitational radiation worked properly as intended.

Then finally, since we are in the Bondi gauge, we found the gravitational news to be

$$
\mathcal{N}_{+}=\frac{1}{2} \operatorname{Re}\left(c_{1} i v \exp (i v u)\right)\left(\sqrt{-(l-1) L_{2}(l+2)}\right)_{2} Z_{l m}
$$


which then further simplify to

$$
\mathcal{N}_{+}=\operatorname{Re}(-0.3778509291 m-0.5950899448 \mathrm{im}),
$$

with the Bondi mass loss of $0.0114 \mathrm{~m}^{2}$. The author in [24] has done a similar work for a single point particle in close orbit around a Schwarzschild black hole in the Bondi-frame and obtained the Bondi mass loss of $0.00089897 \mathrm{~m}^{2}$. $\mathrm{He}$ succeeded in validating the results by comparing it with that of the $5.5 \mathrm{PN}$ formalism by Poisson [25] and Sasaki et al. [26] for the same problem. Thus the methods used in this article open up the possibilities in numerical relativity to be able to study analytically the gravitational radiation emitted by a systems consisting of one black hole and two equal orbiting black holes/neutron stars. With further improvement, the method can be develop to look at two unequal orbiting black holes or neutron stars or a combination of both with efficiency and accuracy as demonstrated in [24] for single orbiting black hole/neutron star.

\section{Acknowledgements}

I would like to thank Professor Nigel Bishop for suggestions and validation of the numerical methods and results and on improving the manuscript. I would also like to thank the National Research Foundation of South Africa under GUN 2053724 for financial support.

\section{References}

[1] Baumgarte, T.W., Skoge, M.L. and Shopiro, S.L. (2004) Physical Review D, 70, Article ID: 064040. https://doi.org/10.1103/PhysRevD.70.064040

[2] Carter, B. and Luminet, J.P. (1983) Astronomy \& Astrophysics, 121, 97-113.

[3] Carter, B. and Luminet, J.P. (1985) Astronomy \& Astrophysics, 121, 23.

[4] Chandrasekhar, S. (1969) Ellipsoidal Figures of Equilibrium. Yale University Press, New Heaven.

[5] Faber, J.A., Baumgarte, T.W., Shapiro, S.L. and Taniguchi, K. (2006) The Astrophysical Journal, 641, L93. https://doi.org/10.1086/504111

[6] Faber, J.A., Baumgarte, T.W., Shapiro, S.L., Taniguchi, K. and Rasio, F.A. (2006) Physical Review D, 73, Article ID: 024012. https://doi.org/10.1103/PhysRevD.73.024012

[7] Fishbone, L.G. (1973) The Astrophysical Journal, 185, 43-67. https://doi.org/10.1086/152395

[8] Grandclément, P. (2006) Physical Review D, 74, Article ID: 124002. https://doi.org/10.1103/PhysRevD.74.124002

[9] Grandclément, P. (2007) Physical Review D, 75, Article ID: 129903(E).

[10] Ishii, M., Shibata, M. and Mino, Y. (2005) Physical Review D, 71, Article ID: 044017. https://doi.org/10.1103/PhysRevD.71.044017

[11] Kobayashi, S., Laguna, P., Phinney, E.S. and Mészáros, P. (2004) The Astrophysical Journal, 615, 855-865. https://doi.org/10.1086/424684

[12] Lai, D. and Wiseman, A.G. (1996) Physical Review D, 54, 3958-3964. https://doi.org/10.1103/PhysRevD.54.3958 
[13] Lee, W.H. (2000) Monthly Notices of the Royal Astronomical Society, 318, 606-624. https://doi.org/10.1046/j.1365-8711.2000.03870.x

[14] Löffler, F., Rezzollas and Ansorg, M. (2006) Physical Review D, 74, Article ID: 104018. https://doi.org/10.1103/PhysRevD.74.104018

[15] Mashhoon, B. (1975) The Astrophysical Journal, 197, 705-716. https://doi.org/10.1086/153560

[16] Miller, M.C. (2005) The Astrophysical Journal, 626, L41-L44. https://doi.org/10.1086/431583

[17] Postnov, K.A. and Yungelson, L.R. (2006) Living Reviews in Relativity, 9, 6. http://www.livingreviews.org/lrr-2006-6

[18] Rosswog, S., Speith, R. and Wynn, G.A. (2004) Monthly Notices of the Royal Astronomical Society, 351, 1121-1133. https://doi.org/10.1111/j.1365-2966.2004.07865.x

[19] Shibata, M. and Uryū, K. (2006) Physical Review D, 74, Article ID: 121503(R).

[20] Shibata, M. and Uryū, K. (2007) Classical and Quantum Gravity, 24, S125-S137. https://doi.org/10.1088/0264-9381/24/12/S09

[21] Sopuerta, C.F., Sperhake, U. and Laguna, P. (2006) Classical and Quantum Gravity, 23, S579-S598. https://doi.org/10.1088/0264-9381/23/16/S15

[22] Taniguchi, K., Baumgarte, T.W., Faber, J.A. and Shapiro, S.L. (2006) Physical Review $D, 74$, Article ID: 041502(R).

[23] Taniguchi, K., Baumgarte, T.W., Faber, J.A. and Shapiro, S.L. (2007) Physical Review $D$, 75, Article ID: 084005. https://doi.org/10.1103/PhysRevD.75.084005

[24] Kubeka, A.S. (2013) Applications of Linearized Perturbations of the Schwarzschild Geometry within the Bondi-Sachs Formalism. PHD Thesis.

[25] Poisson, E. (1993) Physical Review D, 47, 1497-1510. https://doi.org/10.1103/PhysRevD.47.1497

[26] Sasaki, M. and Tagoshi, H. (2003) Living Reviews in Relativity, 6, 6. https://doi.org/10.12942/lrr-2003-6

[27] Bishop, N.T. (2005) Classical and Quantum Gravity, 22, 2393-2406.

[28] Bondi, H., van der Burg, M.J.G. and Metzner, A.W.K. (1962) Proceedings of the Royal Society of London A, 269, 21-52. https://doi.org/10.1098/rspa.1962.0161

[29] Sachs, R.K. (1963) Physical Review Letters, 150, 66.

[30] Bishop, N.T., Gómez, R., Lehner, L., Maharaj, M. and Winicour, J. (1997) Physical Review D, 56, 6298-6309. https://doi.org/10.1103/PhysRevD.56.6298

[31] Bishop, N.T. and Kubeka, A.S. (2009) Physical Review D, 80, Article ID: 064011. https://doi.org/10.1103/PhysRevD.80.064011 


\section{Appendix}

1) The constraints computed at $r_{0}$

$$
\begin{aligned}
R_{\text {uu- }}\left(r_{0}\right)= & .000000000 \times 10^{-9}\left(-200 r_{0}^{9} c_{7}+6.005358575 \times 10^{19} i c_{7} r_{0}^{3}\right. \\
& +6.8000000 \times 10^{7} i r_{0}^{7} c_{10}+1.292409115 \times 10^{16} i \ln \left(r_{0}\right) c_{7} r_{0}^{4} \\
& -3.000000000 \times 10^{9} c_{10} r_{0}^{6}-6.692070654 \times 10^{17} c_{7} r_{0}^{5} \\
& -1.440000000 \times 10^{11} r_{0}^{5} c_{9}-1000 i c_{7} r_{0}^{8} \\
& -1.224000000 \times 10^{9} i c_{9} r_{0}^{7}+2.72000000 \times 10^{8} i r_{0}^{5} c_{10} \\
& +4.896000000 \times 10^{9} i r_{0}^{4} c_{6}-3.227234492 \times 10^{19} i c_{7} r_{0}^{2} \\
& +2.084590843 \times 10^{16} c_{7} r_{0}^{7}-4.896000000 \times 10^{9} i c_{9} r_{0}^{6} \\
& +7.215316909 \times 10^{17} \ln \left(r_{0}\right) c_{7} r_{0}^{5}+1.044710385 \times 10^{19} i r_{0}^{4} c_{7} \\
& -1000 r_{0}^{8} c_{7}+1.884955592 \times 10^{11} r_{0}^{8} \rho \\
& -3.600000000 \times 10^{10} r_{0}^{7} c_{9}+8.16000000 \times 10^{8} i r_{0}^{7} c_{6} \\
& -3.418052808 \times 10^{9} i r_{0}^{7} \rho-6.836105613 \times 10^{9} i r_{0}^{8} \rho \\
& +4.488000000 \times 10^{9} i r_{0}^{6} c_{6}+3.015928948 \times 10^{11} r_{0}^{8} \rho \\
& +7.179615196 \times 10^{17} c_{7} r_{0}^{4}+1.098242223 \times 10^{19} c_{7} r_{0}^{3} \\
& +20000 i r_{0}^{9} c_{7}+2.72000000 \times 10^{8} i r_{0}^{6} c_{10} \\
& +1.806026323 \times 10^{17} \ln \left(r_{0}\right) c_{7} r_{0}^{6}-2.163898247 \times 10^{17} i c_{7} r_{0}^{6} \\
& +8.160000000 \times 10^{9} i r_{0}^{5} c_{6}+7.212387448 \times 10^{17} \ln \left(r_{0}\right) c_{7} r_{0}^{4} \\
& +8.346481884 \times 10^{16} c_{7} r_{0}^{6}-1.507964474 \times 10^{11} r_{0}^{6} \rho \\
& +2.111184000 \times 10^{19} c_{7}-1.20000000 \times 10^{10} c_{10} r_{0}^{5} \\
& +1.382918067 \times 10^{18} i c_{7} r_{0}^{5}-1.311705542 \times 10^{16} i r_{0}^{6} \ln \left(r_{0}\right) c_{7} \\
& -4.896000000 \times 10^{9} i c_{9} r_{0}^{5}-4.087019553 \times 10^{15} i r_{0}^{7} \ln \left(r_{0}\right) c_{7} \\
& +1.741939934 \times 10^{19} c_{7} r_{0}+7.323651653 \times 10^{13} r_{0}^{7} \ln \left(r_{0}\right) c_{7} \\
& -6.491754249 \times 10^{20} i c_{7} r_{0}-1.440000000 \times 10^{11} r_{0}^{6} c_{9} \\
& -4.113828618 \times 10^{16} i c_{7} r_{0}^{7}-2.563539606 \times 10^{9} i r_{0}^{9} p \\
& +1.650271349 \times 10^{19} c_{7} r_{0}^{2}-3.42398706 \times 10^{15} i r_{0}^{5} \ln \left(r_{0}\right) c_{7} \\
& \left.-8.031498194 \times 10^{20} i c_{7}-1.200000000 \times 10^{10} c_{10} r_{0}^{4}\right) /\left(r_{0}^{7}\left(r_{0}+2\right)^{2}\right) \\
&
\end{aligned}
$$

$$
\begin{aligned}
R_{u u+}\left(r_{0}\right)= & 1 / 2\left(( r _ { 0 } ^ { 2 } - 2 r _ { 0 } ) \left(-915.9586340 i c_{2} / r_{0}^{3}+0.8160000000 i c_{4}\right.\right. \\
& -0.6052309472 c_{2}+6745.674492 c_{2} / r_{0}^{7} \\
& +80948.09432 c_{2} / r_{0}^{5}+7518.667272 i c_{2} / r_{0}^{4} \\
& +90224.00728 i c_{2} / r_{0}^{5}+1.503112547 i c_{2}-69.78185262 i c_{2} / r_{0}^{2} \\
& \left.-2733.489212 c_{2} / r_{0}^{3}+12 c_{1} / r_{0}^{3}+36.07819002 c_{2} / r_{0}^{2}\right) \\
& -7518.667272 i c_{2} / r_{0}^{2}-53.40273063 i c_{2} r_{0} \\
& +0.1360 i r_{0}\left(1253.111212 i c_{2} / r_{0}^{2}-457.9793170 i c_{2} / r_{0}\right. \\
& +7518.667272 i c_{2} / r_{0}^{3}-0.3026154736 c_{2} r_{0}^{2} \\
& +22.10459629 c_{2} r_{0}+1124.279082 c_{2} / r_{0}^{2} \\
& +6745.674528 c_{2} / r_{0}^{3}+12 r_{0} c_{4}+8.900455105 i c_{2} r_{0}
\end{aligned}
$$




$$
\begin{aligned}
& +69.78185262 i \ln \left(r_{0}\right) c_{2}+0.7515562734 i c_{2} r_{0}^{2} \\
& +0.4080000000 i r_{0}^{2} c_{4}-1366.744606 c_{2} / r_{0} \\
& \left.+6 c_{1} / r_{0}-36.07819002 \ln \left(r_{0}\right) c_{2}+c_{5}\right)+1.815692842 c_{2} r_{0}^{2} \\
& -132.6275777 c_{2} r_{0}-6745.674492 c_{2} / r_{0}^{2} \\
& -40474.04717 c_{2} / r_{0}^{3}-72 r_{0} c_{4}+2747.875902 i c_{2} / r_{0} \\
& -45112.00363 i c_{2} / r_{0}^{3}-418.6911157 i \ln \left(r_{0}\right) c_{2} \\
& -4.509337640 i c_{2} r_{0}^{2}+8200.467636 c_{2} / r_{0} \\
& -36 c_{1} / r_{0}+216.4691401 \ln \left(r_{0}\right) c_{2}-6 c_{5} \\
& +12 r_{0}\left(0.5043591226 \times 10^{-1} c_{2}-2.568292000 \times 10^{-11} c_{2} / r_{0}\right. \\
& -9.800840000 \times 10^{-11} i c_{2} / r_{0}-50.69289058 c_{2} / r_{0}^{2} \\
& -0.680 \times 10^{-1} i c_{4}+201.0479721 i c_{2} / r_{0}^{3} \\
& -0.1252593789 i c_{2}-6745.674528 c_{2} / r_{0}^{5} \\
& +228.5542745 c_{2} / r_{0}^{3}+1687.678839 i c_{2} / r_{0}^{7} \\
& -38.40352765 i c_{2} / r_{0}^{2}-7518.667272 i c_{2} / r_{0}^{5} \\
& \left.+2 c_{1} / r_{0}^{2}+2 c_{1} / r_{0}^{3}+2614.546974 c_{2} / r_{0}^{4}\right) \\
& +0.8160 i r_{0}^{3}\left(0.5043591226 \times 10^{-1} c_{2}-2.568292000 \times 10^{-11} c_{2} / r_{0}\right. \\
& -9.800840000 \times 10^{-11} i c_{2} / r_{0}-50.69289058 c_{2} / r_{0}^{2} \\
& -0.680 \times 10^{-1} i c_{4}+201.0479721 i c_{2} / r_{0}^{3}-0.1252593789 i c_{2} \\
& -6745.674528 c_{2} / r_{0}^{5}+228.5542745 c_{2} / r_{0}^{3}+1687.678839 i c_{2} / r_{0}^{4} \\
& -38.40352765 i c_{2} / r_{0}^{2}-7518.667272 i c_{2} / r_{0}^{5}+2 c_{1} / r_{0}^{2} \\
& \left.+2 c_{1} / r_{0}^{3}+2614.546974 c_{2} / r_{0}^{4}-2.448000000 i r_{0}^{2} c_{4}\right) / r_{0}^{3} \\
& -20
\end{aligned}
$$

$$
\begin{aligned}
R_{u r-}\left(r_{0}\right)= & 1.111111111 \times 10^{-9}\left(-1.272774256 \times 10^{19} i c_{7} r_{0}^{2}\right. \\
& +1.314989772 \times 10^{20} i c_{7} r_{0}-1.589998513 \times 10^{18} i c_{7} r_{0}^{3} \\
& +76 i c 7 r_{0}^{6}+3.011811822 \times 10^{20} i c_{7} \\
& +4.846534181 \times 10^{14} i c_{7} r_{0}^{4}+40952 i r_{0}^{5} c_{7} \\
& -7.916940003 \times 10^{18} c_{7}+2.280721975 \times 10^{17} c_{7} r_{0}^{2} \\
& -3.616361705 \times 10^{18} c_{7} r_{0}+8.260193057 \times 10^{16} c_{7} r_{0}^{3} \\
& -3.02160 \times 10^{5} c_{7} r_{0}^{5}-1.51080 \times 10^{5} c_{7} r_{0}^{6} \\
& +3.392920066 \times 10^{10} r_{0}^{6} \rho \\
& \left.+2.704645291 \times 10^{16} c_{7} r_{0}^{4}\right) /\left(r_{0}^{6}\left(r_{0}+2\right)\right),
\end{aligned}
$$

$$
\begin{aligned}
R_{u r+}\left(r_{0}\right)= & 1 / 2\left(r _ { 0 } \left(-915.9586340 i c_{2} / r_{0}^{3}+0.8160000000 i c_{4}\right.\right. \\
& -0.6052309472 c_{2}+6745.674492 c_{2} / r_{0}^{4} \\
& +80948.09432 c_{2} / r_{0}^{5}+7518.667272 i c_{2} / r_{0}^{4} \\
& +90224.00728 i c_{2} / r_{0}^{5}+1.503112547 i c_{2} \\
& -69.78185262 i c_{2} / r_{0}^{2}-2733.489212 c_{2} / r_{0}^{3}+12 c_{1} / r_{0}^{3}
\end{aligned}
$$




$$
\begin{aligned}
& \left.+36.07819002 c_{2} / r_{0}^{2}\right)+6 r_{0}^{2}\left(2.568292000 \times 10^{-11} c_{2} / r_{0}^{2}\right. \\
& +9.800840000 \times 10^{-11} i c_{2} / r_{0}^{2}+101.3857812 c_{2} / r_{0}^{3} \\
& -603.1439163 i c_{2} / r_{0}^{4}+33728.37264 c_{2} / r_{0}^{6} \\
& -685.6628235 c_{2} / r_{0}^{4}-6750.715356 i c_{2} / r_{0}^{5} \\
& +76.80705530 i c_{2} / r_{0}^{3}+37593.33636 i c_{2} / r_{0}^{6} \\
& \left.-4 c_{1} / r_{0}^{3}-6 c_{1} / r_{0}^{4}-10458.18790 c_{2} / r_{0}^{5}\right) \\
& +12 r_{0}\left(0.5043591226 \times 10^{-1} c_{2}-2.568292000 \times 10^{-11} c_{2} / r_{0}\right. \\
& -9.800840000 \times 10^{-11} i c_{2} / r_{0}-50.69289058 c_{2} / r_{0}^{2} \\
& -0.680 \times 10^{-1} i c_{4}+201.0479721 i c_{2} / r_{0}^{3} \\
& -0.1252593789 i c_{2}-6745.674528 c_{2} / r_{0}^{5} \\
& +228.5542745 c_{2} / r_{0}^{3}+1687.678839 i c_{2} / r_{0}^{4} \\
& -38.40352765 i c_{2} / r_{0}^{2}-7518.667272 i c_{2} / r_{0}^{5} \\
& \left.\left.+2 c_{1} / r_{0}^{2}+2 c_{1} / r_{0}^{3}+2614.546974 c_{2} / r_{0}^{4}\right)\right) / r_{0}^{2},
\end{aligned}
$$

$$
\begin{aligned}
q^{A} R_{u A-}\left(r_{0}\right)= & 1.000000000 \times 10^{-10}\left(16000 i c_{7} r_{0}^{8}-1.000000 \times 10^{6} r_{0}^{8} c_{7}\right. \\
& +6.95123928 \times 10^{18} c_{7} r_{0}^{4}+4.60818516 \times 10^{20} i c_{7} r_{0} \\
& +6.811699261 \times 10^{15} i c_{7} r_{0}^{7}+1.202064574 \times 10^{18} \ln \left(r_{0}\right) c_{7} r_{0}^{4} \\
& +1.140199529 \times 10^{19} i c_{7} r_{0}^{5}-7.391764651 \times 10^{20} i c_{7} r_{0}^{3} \\
& -1.220608500 \times 10^{14} c_{7} r_{0}^{7}+4.461943440 \times 10^{21} i c_{7} \\
& +300 r_{0}^{7} p+5.385037980 \times 10^{15} i \ln \left(r_{0}\right) c_{7} r_{0}^{6} \\
& -2.000000000 \times 10^{10} c_{10} r_{0}^{4}+2.154015192 \times 10^{16} i \ln \left(r_{0}\right) c_{7} r_{0}^{5} \\
& -2.671516126 \times 10^{21} i c_{7} r_{0}^{2}+2.040000000 \times 10^{9} i r_{0}^{6} c_{6} \\
& +3.005161436 \times 10^{17} \ln \left(r_{0}\right) c_{7} r_{0}^{6}+8.160000000 \times 10^{9} i r_{0}^{4} c_{6} \\
& -2.000000000 \times 10^{10} c_{10} r_{0}^{5}-5.000000000 \times 10^{9} c_{10} r_{0}^{6} \\
& +1.202064574 \times 10^{18} \ln \left(r_{0}\right) c_{7} r_{0}^{5}-3.817318694 \times 10^{18} c_{7} r_{0}^{5} \\
& -6.543021412 \times 10^{16} i r_{0}^{6} c_{7}+8.809405089 \times 10^{19} c_{7} r_{0}^{3} \\
& +1.455381063 \times 10^{20} c_{7} r_{0}^{2}+2.154015192 \times 10^{16} i \ln \left(r_{0}\right) c_{7} r_{0}^{4} \\
& -1.44797289 \times 10^{19} c_{7} r_{0}+8.160000000 \times 10^{9} i r_{0}^{5} c_{6} \\
& +4.272566009 \times 10^{9} i r_{0}^{9} \rho-3.509551026 \times 10^{17} c_{7} r_{0}^{6} \\
& +1.001418528 \times 10^{20} i r_{0}^{4} c_{7}-1.172880000 \times 10^{20} c_{7} \\
& \left.+8.545132018 \times 10^{9} i r_{0}^{8} \rho\right) /\left(r_{0}^{6}\left(r_{0}+2\right)^{2}\right),
\end{aligned}
$$

$$
\begin{aligned}
q^{A} R_{u A+}\left(r_{0}\right)= & 1 / 2\left(-1253.111212 i c_{2} / r_{0}^{2}-c_{5}-1124.279082 c_{2} / r_{0}^{2}\right. \\
& +0.3026154736 c_{2} r_{0}^{2}-22.10459629 c_{2} r_{0}-12 r_{0} c_{4} \\
& +36.07819002 \ln \left(r_{0}\right) c_{2}-8 r_{0}^{2}\left(2.568292000 \times 10^{-11} c_{2} / r_{0}^{2}\right. \\
& +9.800840000 \times 10^{-11} i c_{2} / r_{0}^{4}+101.3857812 c_{2} / r_{0}^{3} \\
& -603.1439163 i c_{2} / r_{0}^{4}+33728.37264 c_{2} / r_{0}^{6}
\end{aligned}
$$




$$
\begin{aligned}
& -685.6628235 c_{2} / r_{0}^{4}-6750.715356 i c_{2} / r_{0}^{5} \\
& +76.80705530 i c_{2} / r_{0}^{3}+37593.33636 i c_{2} / r_{0}^{6} \\
& \left.-4 c_{1} / r_{0}^{3}-6 c_{1} / r_{0}^{4}-10458.18790 c_{2} / r_{0}^{5}\right) \\
& +r_{0}\left(457.9793170 i c_{2} / r_{0}^{2}+0.8160000000 i r_{0} c_{4}\right. \\
& +8.900455105 i c_{2}-0.6052309472 c_{2} r_{0}+22.10459629 c_{2} \\
& -2248.558164 c_{2} / r_{0}^{3}-20237.02358 c_{2} / r_{0}^{4}+12 c_{4} \\
& -2506.222424 i c_{2} / r_{0}^{3}-22556.00182 i c_{2} / r_{0}^{4} \\
& +1.503112547 i c_{2} r_{0}+69.78185262 i c_{2} / r_{0} \\
& \left.+1366.744606 c_{2} / r_{0}^{2}-6 c_{1} / r_{0}^{2}-36.07819002 c_{2} / r_{0}\right) \\
& +4 r_{0}^{3}\left(2.568292000 \times 10^{-11} c_{2} / r_{0}^{2}\right. \\
& +9.800840000 \times 10^{-11} i c_{2} / r_{0}^{2}+101.3857812 c_{2} / r_{0}^{3} \\
& -603.1439163 i c_{2} / r_{0}^{4}+33728.37264 c_{2} / r_{0}^{6} \\
& -685.6628235 c_{2} / r_{0}^{4}-6750.715356 i c_{2} / r_{0}^{5} \\
& +76.80705530 i c_{2} / r_{0}^{3}+37593.33636 i c_{2} / r_{0}^{6} \\
& \left.-4 c_{1} / r_{0}^{3}-6 c_{1} / r_{0}^{4}-10458.18790 c_{2} / r_{0}^{5}\right) \\
& +r_{0}^{4}\left(-5.136584000 \times 10^{-11} c_{2} / r_{0}^{3}\right. \\
& -1.960168000 \times 10^{-10} i c_{2} / r_{0}^{3}-304.1573436 c_{2} / r_{0}^{4} \\
& +2412.575665 i c_{2} / r_{0}^{5}-2.023702358 \times 10^{5} c_{2} / r_{0}^{7} \\
& +2742.651294 c_{2} / r_{0}^{5}+33753.57678 i c_{2} / r_{0}^{6} \\
& -230.4211659 i c_{2} / r_{0}^{4}-2.255600182 \times 10^{5} i c_{2} / r_{0}^{7} \\
& \left.+12 c_{1} / r_{0}^{7}+24 c_{1} / r_{0}^{5}+52290.93950 c_{2} / r_{0}^{6}\right) \\
& +2 r_{2}^{2}\left(0.5043591226 \times 10^{-1} c_{2}-2.568292000 \times 10^{-11} c_{2} / r_{0}\right. \\
& -9.800840000 \times 10^{-11} i c_{2} / r_{0}-50.69289058 c_{2} / r_{0}^{2} \\
& -0.680 \times 10^{-1} i c_{4}+201.0479721 i c_{2} / r_{0}^{3} \\
& -0.1252593789 i c_{2}-6745.674528 c_{2} / r_{0}^{5} \\
& +228.5542745 c_{2} / r_{0}^{3}+1687.678839 i c_{2} / r_{0}^{4} \\
& -38.40352765 i c_{2} / r_{0}^{2}-7518.667272 i c_{2} / r_{0}^{5} \\
& \left.+2 c_{1} / r_{0}^{2}+2 c_{1} / r_{0}^{3}+2614.546974 c_{2} / r_{0}^{4}\right) \\
& -8.900455105 i c_{2} r_{0}-0.2720 i r_{0}^{2}\left(c_{4}+c_{1} / r_{0}\right. \\
& +c_{2}\left(1+(13.04336905144130-1.31528646137769 i)\left(1 / r_{0}-1 / 6\right)\right. \\
& +(77.34402850-15.99899824 i)\left(1 / r_{0}-1 / 6\right)^{2} \\
& \left.\left.+(-187.3798480-208.8518687 i)\left(1 / r_{0}-1 / 6\right)^{3}\right)\right) \\
& +457.9793170 i c_{2} / r_{0} \\
& -0.680 \times 10^{-1} i r_{0}^{4}\left(2.568292000 \times 10^{-11} c_{2} / r_{0}^{2}\right. \\
& +9.800840000 \times 10^{-11} i c_{2} / r_{0}^{2}+101.3857812 c_{2} / r_{0}^{2}
\end{aligned}
$$




$$
\begin{aligned}
& -603.1439163 i c_{2} / r_{0}^{4}+33728.37264 c_{2} / r_{0}^{6} \\
& -685.6628235 c_{2} / r_{0}^{4}-6750.715356 i c_{2} / r_{0}^{5} \\
& +76.80705530 i c_{2} / r_{0}^{3}+37593.33636 i c_{2} / r_{0}^{6} \\
& \left.-4 c_{1} / r_{0}^{3}-6 c_{1} / r_{0}^{4}-10458.18790 c_{2} / r_{0}^{5}\right) \\
& -7518.667272 i c_{2} / r_{0}^{3}-69.78185262 i \ln \left(r_{0}\right) c_{2} \\
& -0.7515562734 i c_{2} r_{0}^{2}-2 r_{0}^{3}\left(-5.136584000 \times 10^{-11} c_{2} / r_{0}^{3}\right. \\
& -1.960168000 \times 10^{-10} i c_{2} / r_{0}^{3}-304.1573436 c_{2} / r_{0}^{4} \\
& +2412.575665 i c_{2} / r_{0}^{5}-2.023702358 \times 10^{5} c_{2} / r_{0}^{7} \\
& +2742.651294 c_{2} / r_{0}^{5}+33753.57678 i c_{2} / r_{0}^{6} \\
& -230.4211659 i c_{2} / r_{0}^{4}-2.255600182 \times 10^{5} i c_{2} / r_{0}^{7} \\
& \left.+12 c_{1} / r_{0}^{4}+24 c_{1} / r_{0}^{5}+52290.93950 c_{2} / r_{0}^{6}\right) \\
& -0.4080000000 i r_{0}^{2} c_{4}-6745.674528 c_{2} / r_{0}^{3} \\
& \left.+1366.744606 c_{2} / r_{0}-6 c_{1} / r_{0}\right) / r_{0}^{2},
\end{aligned}
$$

\section{2) The Bondi metric variables computed at $r_{0}$}

$$
\begin{aligned}
U_{+}\left(r_{0}\right)= & 0.5043591226 \times 10^{-1} c_{2}-2.568292000 \times 10^{-11} c_{2} / r_{0} \\
& -9.800840000 \times 10^{-11} i c_{2} / r_{0}-50.69289058 c_{2} / r_{0}^{2} \\
& -0.680 \times 10^{-1} i c_{4}+201.0479721 i c_{2} / r_{0}^{3} \\
& -0.1252593789 i c_{2}-6745.674528 c_{2} / r_{0}^{5} \\
& +228.5542745 c_{2} / r_{0}^{3}+1687.678839 i c_{2} / r_{0}^{4} \\
& -38.40352765 i c_{2} / r_{0}^{2}-7518.667272 i c_{2} / r_{0}^{5} \\
& +2 c_{1} / r_{0}^{2}+2 c_{1} / r_{0}^{3}+2614.546974 c_{2} / r_{0}^{4}
\end{aligned}
$$

$$
\begin{aligned}
U_{-}\left(r_{0}\right)= & \left(4.887000002 \times 10^{7}-1.859143100 \times 10^{9} i\right) c_{7} / r_{0}^{5} \\
& +4 c_{7}\left(2.865551000 \times 10^{6}-1.523351300 \times 10^{7} i\right. \\
& +\left(4.887000002 \times 10^{7}-1.859143100 \times 10^{9} i\right)\left(1 / r_{0}-1 / 6\right) / r_{0}^{4} \\
& +\left(-2.443500001 \times 10^{7}+9.295715500 \times 10^{8} i\right) c_{7} / r_{0}^{4} \\
& +\left(2 \left(c_{6}+c_{7}\left(29144-2.280672000 \times 10^{5} i\right.\right.\right. \\
& +\left(2.865551000 \times 10^{6}-1.523351300 \times 10^{7} i\right)\left(1 / r_{0}-1 / 6\right) \\
& \left.\left.\left.+\left(2.443500001 \times 10^{7}-9.295715502 \times 10^{8} i\right)\left(1 / r_{0}-1 / 6\right)^{2}\right)\right)\right) / r_{0}^{3} \\
& -c_{7}\left(2.865551000 \times 10^{6}-1.523351300 \times 10^{7} i\right. \\
& \left.+\left(4.887000002 \times 10^{7}-1.859143100 \times 10^{9} i\right)\left(1 / r_{0}-1 / 6\right)\right) / r_{0}^{3} \\
& -\left(0.680 \times 10^{-1} i\right) c_{7}\left(2.865551000 \times 10^{6}-1.523351300 \times 10^{7} i\right. \\
& +\left(4.887000002 \times 10^{7}-1.859143100 \times 10^{9} i\right)\left(1 / r_{0}-1 / 6\right) / r_{0}^{2} \\
& +\left(2 \left(c_{6}+c_{7}\left(29144-2.280672000 \times 10^{5} i\right.\right.\right.
\end{aligned}
$$




$$
\begin{aligned}
+ & \left(2.865551000 \times 10^{6}-1.523351300 \times 10^{7} i\right)\left(1 / r_{0}-1 / 6\right) \\
+ & \left.\left.\left.\left(2.443500001 \times 10^{7}-9.295715502 \times 10^{8} i\right)\left(1 / r_{0}-1 / 6\right)^{2}\right)\right)\right) / r_{0}^{2} \\
+ & \left(0.680 \times 10^{-1} i\right)\left(c_{6}+c_{7}\left(29144-2.280672000 \times 10^{5} i\right.\right. \\
+ & \left(2.865551000 \times 10^{6}-1.523351300 \times 10^{7} i\right)\left(1 / r_{0}-1 / 6\right) \\
+ & \left.\left.\left(2.443500001 \times 10^{7}-9.295715502 \times 10^{8} i\right)\left(1 / r_{0}-1 / 6\right)^{2}\right)\right) / r_{0} \\
- & \left(0.680 \times 10^{-1} i\right)\left(c_{9}+c_{6} / r_{0}+c_{7}(-2518.141205+699.71985 i\right. \\
+ & \left(29144-2.280672000 \times 10^{5} i\right)\left(1 / r_{0}-1 / 6\right) \\
+ & \left(1.432775500 \times 10^{6}-7.616756500 \times 10^{6} i\right)\left(1 / r_{0}-1 / 6\right)^{2} \\
+ & \left.\left.\left(8.145000002 \times 10^{6}-3.098571834 \times 10^{8} i\right)\left(1 / r_{0}-1 / 6\right)^{3}\right)\right) \\
\omega_{+}\left(r_{0}\right)= & (1253.111212 i) c_{2} / r_{0}^{2}-(457.9793170 i) c_{2} / r_{0} \\
& +(7518.667272 i) c_{2} / r_{0}^{3}-0.3026154736 c_{2} r_{0}^{2} \\
& +22.10459629 c_{2} r_{0}+1124.279082 c_{2} / r_{0}^{2} \\
& +6745.674528 c_{2} / r_{0}^{3}+12 r_{0} c_{4}+(8.900455105 i) c_{2} r_{0} \\
& +(69.78185262 i)) \ln \left(r_{0}\right) c_{2}+(0.7515562734 i) c_{2} r_{0}^{2} \\
& +(0.4080000000 i) r_{0}^{2} c_{4}-1366.744606 c_{2} / r_{0} \\
& +6 c_{1} / r_{0}-36.07819002 \ln \left(r_{0}\right) c_{2}+c_{5},
\end{aligned}
$$

and

$$
\begin{aligned}
\omega_{-}\left(r_{0}\right)= & -(2156.071282 i) c_{7} r_{0}^{2}-50.26548247 r_{0}^{2} \rho /\left(1+2 / r_{0}\right) \\
& +6 c_{6} / r_{0}-5.147566058 \times 10^{5} c_{7} r_{0}^{2}-63413.86124 c_{7} r_{0} \\
& -2.932200001 \times 10^{8} c_{7} / r_{0}^{3}-4.886999995 \times 10^{7} c_{7} / r_{0}^{2} \\
& -2.356233015 \times 10^{8} c_{7} / r_{0}-6.010322872 \times 10^{7} \ln \left(r_{0}\right) c_{7} \\
& +12 r_{0} c_{9}+\left(1.513990017 \times 10^{7} i\right) c_{7} r_{0} \\
& -\left(1.031580813 \times 10^{9} i\right) c_{7} / r_{0}+\left(1.859143099 \times 10^{9} i\right) c_{7} / r_{0}^{2} \\
& +\left(1.115485860 \times 10^{10} i\right) c_{7} / r_{0}^{3}+(0.4080000000 i) r_{0}^{2} c_{9} \\
& -\left(1.077007596 \times 10^{6} i\right) \ln \left(r_{0}\right) c_{7}+c_{10},
\end{aligned}
$$

The computed constants

$$
\begin{aligned}
c_{5}= & -5.925925926 \times 10^{-21}\left(2.257876807 \times 10^{25} i c_{2} r_{0}\right. \\
& +1.177568763 \times 10^{22} i \ln \left(r_{0}\right) c_{2} r_{0}^{4}-2.537550206 \times 10^{25} i c_{2} \\
& -1.179321011 \times 10^{22} i c_{2} r_{0}^{4}-6.885000000 \times 10^{19} i r_{0}^{4} c_{1} \\
& -2.276665152 \times 10^{25} c_{2}-1.379990759 \times 10^{20} i c_{2} r_{0}^{5} \\
& +8.836025110 \times 10^{9} c_{2} r_{0}^{6}-6.088194566 \times 10^{21} \ln \left(r_{0}\right) c_{2} r_{0}^{4}
\end{aligned}
$$


$-3.251077700 \times 10^{23} i c_{2} r_{0}^{3}-6.873541974 \times 10^{23} i c_{2} r_{0}^{2}$

$-2.669155863 \times 10^{20} c_{2} r_{0}^{5}+8.950474631 \times 10^{21} c_{2} r_{0}^{4}$

$-4.171449115 \times 10^{23} c_{2} r_{0}^{3}+3.018766507 \times 10^{9} i c_{2} r_{0}^{6}$

$\left.-2.509446061 \times 10^{24} c_{2} r_{0}^{2}+2.174295963 \times 10^{25} c_{2} r_{0}\right) / r_{0}^{4}$,

$c_{7}=\left(-1.683000000 \times 10^{14}+1.416666667 \times 10^{25} i\right) c_{6} /\left(-3.518394608 \times 10^{32}\right.$

$+9.145010221 \times 10^{31} i-1.463828647 \times 10^{23} \ln \left(r_{0}\right)$

$\left.+\left(2.060201741 \times 10^{22} i\right) \ln \left(r_{0}\right)\right)$,

$c_{9}=-1.111111111 \times 10^{-11}\left(1.157988867 \times 10^{17} r_{0}^{8} c_{7}\right.$

$-3.769911183 \times 10^{14} r_{0}^{7} \rho+5.22000 \times 10^{5} r_{0}^{9} c_{7}$

$-8.250022346 \times 10^{21} c_{7} r_{0}^{7}+1.884955592 \times 10^{14} r_{0}^{6} \rho$

$-2.356194490 \times 10^{14} r_{0}^{8} \rho-5.423600000 \times 10^{11} \ln \left(r_{0}\right) c_{7} r_{0}^{5}$

$+9.635910263 \times 10^{22} c_{7} r_{0}^{2}-1.737134306 \times 10^{20} c_{7} r_{0}^{6}$

$+5.0000000 \times 10^{7} i r_{0}^{7} \ln \left(r_{0}\right) c_{7}+6.926736238 \times 10^{21} c_{7} r_{0}^{3}$

$-8.000000 \times 10^{6} i r_{0}^{9} c_{7}-3.240686912 \times 10^{20} c_{7} r_{0}^{5}$

$-1.963050508 \times 10^{24} i c_{7} r_{0}^{2}-2.726124482 \times 10^{19} c_{7} r_{0}^{7}$

$-2.500000 \times 10^{6} r_{0}^{7} \ln \left(r_{0}\right) c_{7}+1.159077064 \times 10^{24} i c_{7} r_{0}$

$-1.143558000 \times 10^{23} c_{7}+2.863090381 \times 10^{20}{i r_{0}^{6}}^{6} c_{7}$

$+2.075034463 \times 10^{15} i c 7 r_{0}^{8}+1.452672443 \times 10^{11} r_{0}^{9} \rho$

$+2.000000000 \times 10^{9} i \ln \left(r_{0}\right) c_{7} r_{0}^{6}-6.319234788 \times 10^{23} i c_{7} r_{0}^{3}$

$+1.495398102 \times 10^{13} i r_{0}^{8} \rho+6.249786891 \times 10^{19} i c 7 r_{0}^{7}$

$+4.350394854 \times 10^{24} i c_{7}-5.000000000 \times 10^{11} \ln \left(r_{0}\right) c_{7} r_{0}^{4}$

$+4.321899263 \times 10^{22} c_{7} r_{0}+6.408849014 \times 10^{12} i r_{0}^{9} \rho$

$-9.236000000 \times 10^{10} \ln \left(r_{0}\right) c_{7} r_{0}^{6}+6.704677816 \times 10^{21} i c_{7} r_{0}^{5}$

$-4.080000000 \times 10^{12} i r_{0}^{6} c_{6}-1.020000000 \times 10^{12} i r_{0}^{7} c_{6}$

$-4.080000000 \times 10^{12} i r_{0}^{5} c_{6}+6.054991092 \times 10^{22} i r_{0}^{4} c_{7}$

$+1.387200000 \times 10^{11} r_{0}^{5} c_{6}+3.468000000 \times 10^{10} r_{0}^{7} c_{6}$

$+7.000000000 \times 10^{9} i \ln \left(r_{0}\right) c_{7} r_{0}^{5}+1.387200000 \times 10^{11} r_{0}^{6} c_{6}$

$+2.500000000 \times 10^{9} i \ln \left(r_{0}\right) c_{7} r_{0}^{4}+4.272566010 \times 10^{12} i r_{0}^{7} \rho$

$\left.+7.263362215 \times 10^{10} r_{0}^{10} \rho\right) /\left(\left(17 i r_{0}^{2}+500 r_{0}^{2}+68 i r_{0}\right.\right.$

$\left.\left.+2000 r_{0}+2000+68 i\right) r_{0}^{5}\right)$,

and

$$
\begin{aligned}
c_{10}= & 2.000000000 \times 10^{-10}\left(-3.509551026 \times 10^{17} c_{7} r_{0}^{6}\right. \\
& +1.202064574 \times 10^{18} \ln \left(r_{0}\right) c_{7} r_{0}^{5}+8.809405089 \times 10^{19} c_{7} r_{0}^{3} \\
& -1.447972890 \times 10^{19} c_{7} r_{0}-3.817318694 \times 10^{18} c_{7} r_{0}^{5} \\
& +1.455381063 \times 10^{20} c_{7} r_{0}^{2}-1.220608500 \times 10^{14} c_{7} r_{0}^{7} \\
& +1.140199529 \times 10^{19} i c_{7} r_{0}^{5}-1.172880000 \times 10^{20} c_{7}
\end{aligned}
$$




$$
\begin{aligned}
& +2.040000000 \times 10^{9} i r_{0}^{6} c_{6}+6.951239280 \times 10^{18} c_{7} r_{0}^{4} \\
& +3.005161436 \times 10^{17} \ln \left(r_{0}\right) c_{7} r_{0}^{6}+4.608185160 \times 10^{20} i c_{7} r_{0} \\
& +4.461943440 \times 10^{21} i c_{7}+2.154015192 \times 10^{16} i \ln \left(r_{0}\right) c_{7} r_{0}^{5} \\
& +8.545132018 \times 10^{9} i r_{0}^{8} \rho+1.202064574 \times 10^{18} \ln \left(r_{0}\right) c_{7} r_{0}^{4} \\
& +1.001418528 \times 10^{20} i r_{0}^{4} c_{7}+16000 i c_{7} r_{0}^{8} \\
& +5.385037980 \times 10^{15} i \ln \left(r_{0}\right) c_{7} r_{0}^{6}+8.160000000 \times 10^{9} i r_{0}^{4} c_{6} \\
& +6.811699261 \times 10^{15} i c_{7} r_{0}^{7}-7.391764651 \times 10^{20} i c_{7} r_{0}^{3} \\
& -2.671516126 \times 10^{21} i c_{7} r_{0}^{2}+2.154015192 \times 10^{16} i \ln \left(r_{0}\right) c_{7} r_{0}^{4} \\
& -1.000000 \times 10^{6} r_{0}^{8} c_{7}+4.272566009 \times 10^{9} i r_{0}^{9} \rho \\
& -6.543021412 \times 10^{16} i r_{0}^{6} c_{7}+8.160000000 \times 10^{9} i r_{0}^{5} c_{6} \\
& \left.+300 r_{0}^{7} \rho\right) /\left(r_{0}^{4}\left(4+4 r_{0}+r_{0}^{2}\right)\right), \\
& c_{1}=(-3.303262047-3.089304784 i) \mathrm{m}, \\
& c_{2}=(-393.9477195-46.65966681 i) \mathrm{m}, \\
& c_{6}=(-394.7245520-8.295859050 i) \mathrm{m},
\end{aligned}
$$

